\title{
TOMOGRAFIA COMPUTADORIZADA NO SEGUIMENTO DE PACIENTES COM TUMORES INTRACRANIANOS
}

\author{
GILBERTO MACHADO DE ALMEIDA* \\ EDUARDO BIANCO ** \\ MILTON $K$. SHIBATA **
}

A importância da tomografia computadorizada (TC) no diagnóstico da maioria dos processos patológicos intracranianos está suficientemente comprovada. Além disto, este método de exame fornece excelente auxílio no acompanhamento de várias entidades, cirúrgicas ou não. Neste trabalho comentamos a tomografia por computador no seguimento de pacientes com tumores cerebrais.

\section{CONTROLE IMEDIATO}

Foi proposto o uso da TC para controlar, durante o ato cirúrgico a remoção de tumores intracranianos. Isto nos parece de pequena utilidade prática, pois a ausência de imagem de tumor na TC não garante que a remoção tenha sido completa. Não vemos como o exame possa detectar a infiltração tumoral. quase sempre presente nos gliomas. Por outro lado, para tumores extracerebrais (meningeomas e neurinomas) o exame não mostra restos tumorais, não vistos com o microscópio. Além disso, o uso da TC durante o ato cirúrgico acarreta vários problemas, que dificultam a atividade da equipe, alongam o tempo operatório e aumentam o risco de infecção.

$\mathrm{Na}$ nossa experiência a TC foi feita imediatamente, apenas com a finalidade de comprovar se $o$ ato cirúrgico havia sido realizado no local exato, como no caso 1:

Caso 1 - J.A.F., 65 anos. Cerca de 4 meses antes da internaç̃o fol notała hemiparesia esquerda discreta que se acentuou há alguns dias. A TC mostrou, na região parietal direita, área discretamente hiperatenuante cercada por edema cerebral. Com contraste houve nitido enhancement" (Fig. 1 A). A angiografia mostrou cesvios caracteristicos de processo expansivo não vascularizado, parietal direito. No ato cirúrgico encontramos edema cerebral e área de amolecimento (comprovada por corte de congelaçăo). Devido a imagem pré-operatória, tão característica de tumor, ficamos com cúvida de termos atingido a regiăo corretar o que foi confirmado por TC realizada imediatamente após (Fig. 1 B).

Clínica Ne-rocirúrgica e Centro de Tomografia do Hospital 9 de Julho, Såo Paulo. Brasil: * Chefe, ** Neurocirurgióes. 


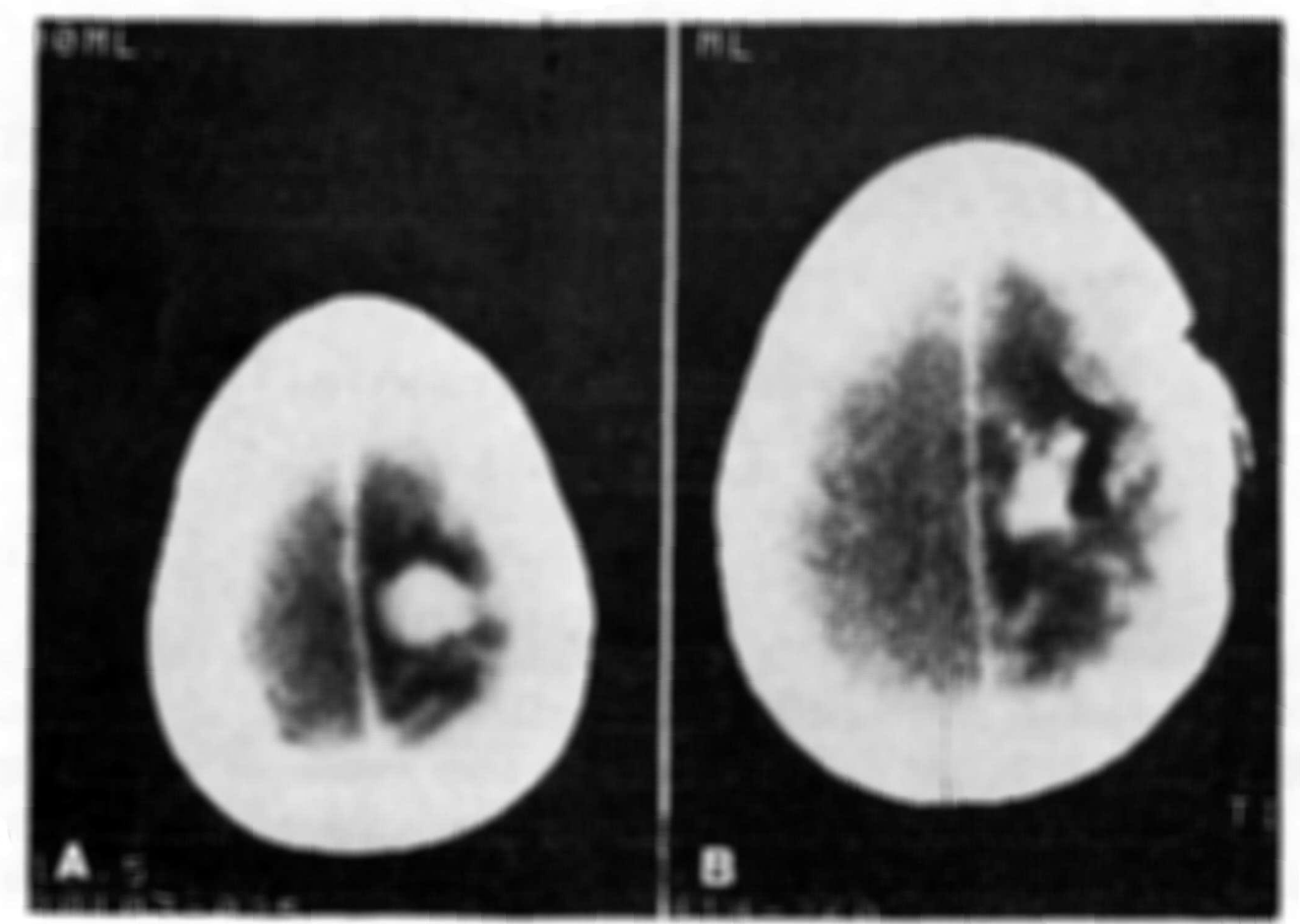

Fig. 1 - Caso 1 - J.A.F. Em A, TC com contraste, mostrando área de retencão, cercada por edema. Em $B, T C$ feita no dia da cirurgia, evidenciando que a região certa havia sido atingida.

\section{CONTROLE PRECOCE}

$\mathrm{O}$ aspecto tomográfico no pós-operatório varia muito dependendo de numerosos fatores, inclusive do tempo decorrido. $O$ exame pode mostrar a presença de ar, sangue, edema, hidrocefalia, cistos, áreas hipoatenuantes de explicação duvidosa. A importância da TC no pós-operatório é nítida quando a evolução não é satisfatória. Este fato trouxe um grave problema para os serviços neurocirúrgicos de hospitais que não possuem o equipamento.

Caso 2 - A.A.L., 4 anos. Este menino tinha im tumor, de fossa posterior (astrocitoma) e grande dilatação ventricular (Fig. 2 A). Foi feita primeiro uma derivação ventrículo-peritoneal e, 7 dias depois, a craniectomia de fossa posterior. No dia seguinte. como a criança estivesse ainda inconsciente, repetimos a TC que evidenciou grandr quantidade de ar fora e dentro dos ventrículos (Fig. 2 B). Foram feitas trepanacões frontais para a retirada de ar. com discreta melhora.

Caso 3 - L.F.G., 28 anos. O paciente apresentava tumor cístico de fossa posterior, confirmado pela angiografia vertebral e TC (Fig. 3 A). A cirurgia mostrou tratar-se de hemangioma cístico. No segundo dia, como o doente permanecia sonolento. foi feita nova tomografia (Fig. $3 \mathrm{~B}$ ), que mostrou área hiperdensa na região operada (sangue?) e bastante ar no espaço subaracnóideo frontal. A presença de ar na região frontal ou nos ventrículos tem-se mostrado constante nos primeiros dias, quando se usa a posição sentała. 


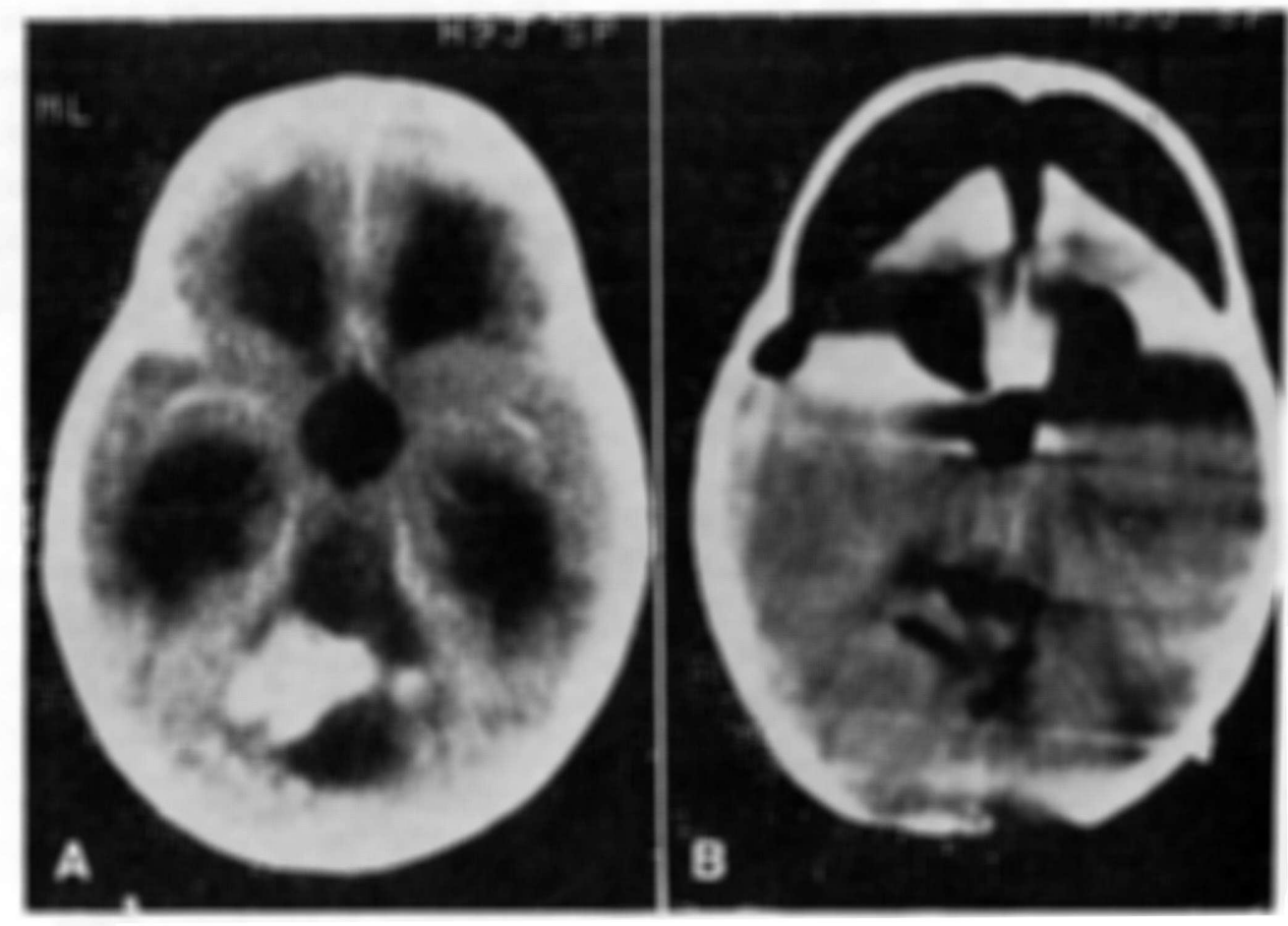

Fig. 2-Caso 2- A.A.L. Em A, TC pré-operatória. com contraste, mostrando o astrocitoma de cerebelo e a dilatacão ventricular. Em B, TC realizada no primeiro dia de posoperatbrio, em que se vê grande quantidade de ar dentro e fora dos ventriculos.
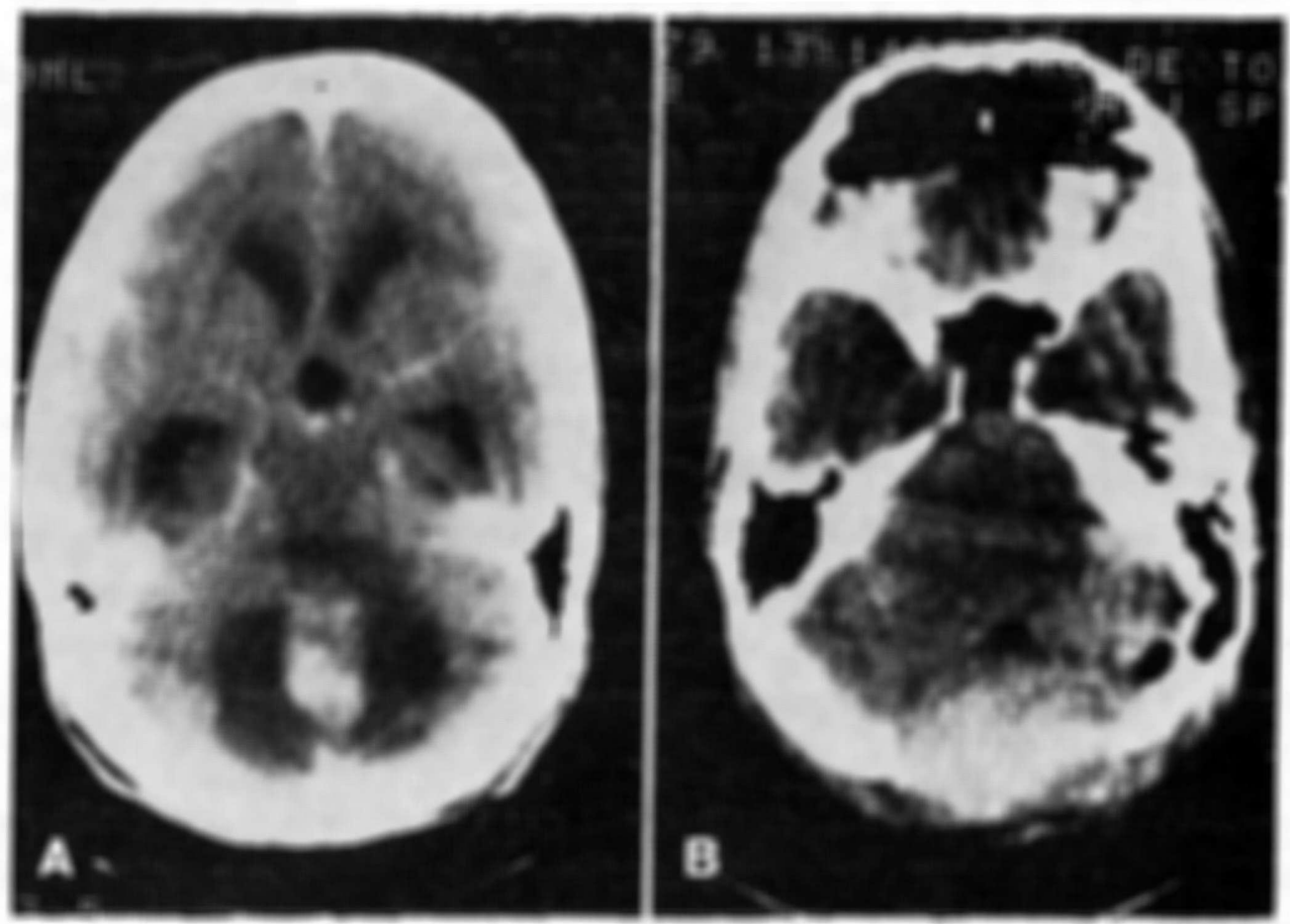

Fig. 3 - Caso 3 . L.F.C. Em A, TC em que se ve hemangioma cistico de fossa posterior. No segundo dia de p6s-operatbrio, nova TC (B) mostra sangue na região operada $e$ ar no espaco subaracnoideo frontal. 
Caso 4 - W.L.P., 16 anos. Este paciente fol operado de um astrocitoma grau II na fossa posterior, que invadia o IV ventrículo. No terceiro dia pós-operatório a TC (Fig. 4 A) mostrava o IV ventriculo ocupado por grande quantidale de sangue. Como o doente estava melhorando, resolvemos aguardar a evolução. Dezoito dias após a cirurgia o sangie estava reabsorvido (Fig. 4 B), persistindo certa dilatação ventric lar. Dois meses após, o aspecto era semelhante. A simples existêricia de sangue na região operada não justifica a reintervenção. preciso analisar vários fatores, principalmente o quadro clínico.
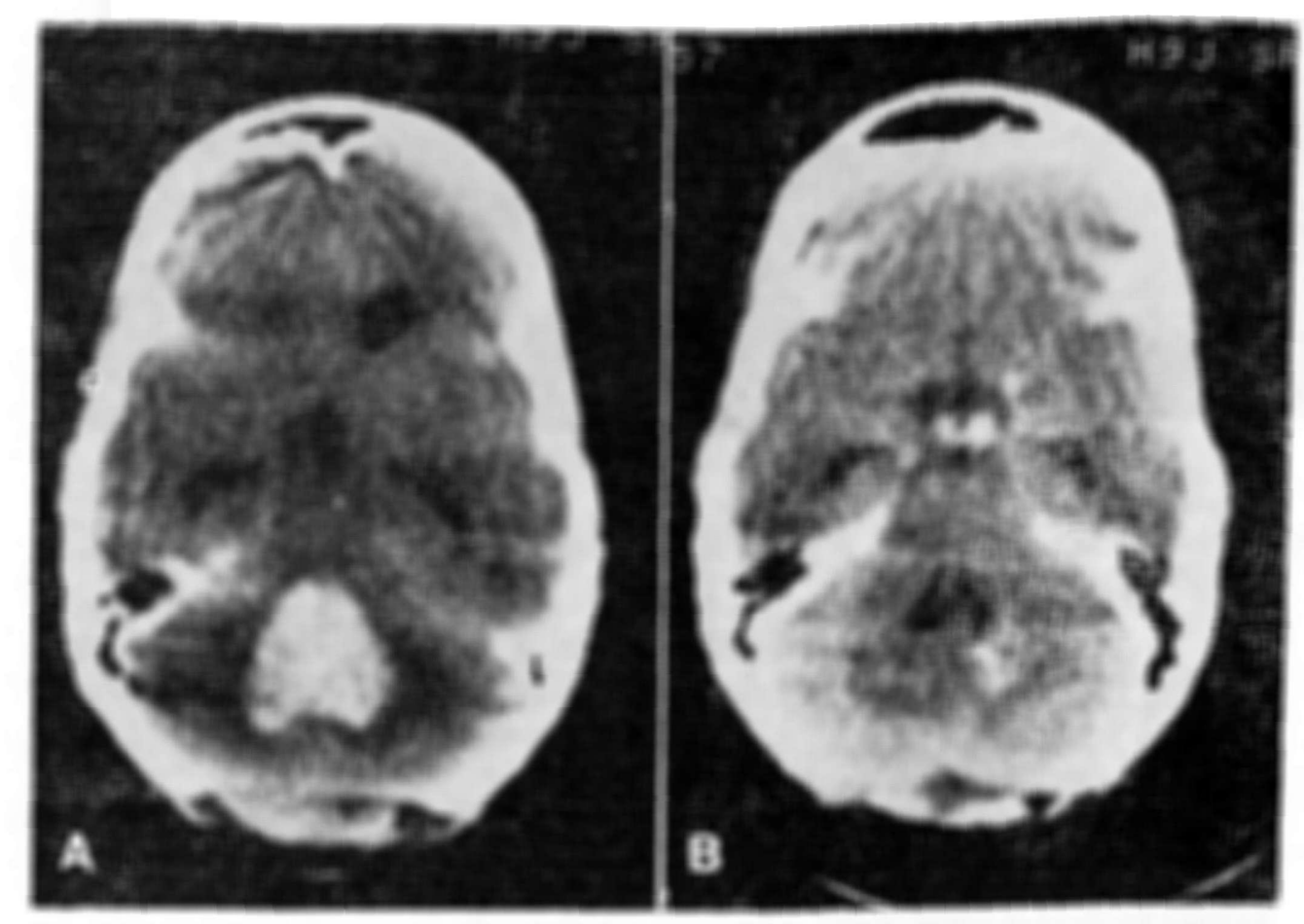

Fig. 4 - Caso 4 . W.L.P. Em A, TC realizada 3 dias apos a cirurgia, mostranto grande quantidade de sangue $200 \mathrm{IV}$ ventriculo. $E m B, T C$ apos 18 dias evidencia que o sangue foi reabsorvido, permanecendo menor dilatacáo do IV ventriculo.

Caso 5 - N.M.S.M.. 61 anos. Este doente tinha apresentado uma convulsão focal há 20 dias. A TC (Fig. 5 A) mostrou tumor frontal esquerdo e a angiografia confirmou a hipótese de meningeoma. No terceiro dia de pós-operatório houve piora do quałro, com sonolência e hemiparesia. Nova TC (Fig. 5 B) mostrou sangue no leito tumoral. $\mathrm{tm}$ pouco de ar e edema. Como a piora havia sido relativamente discreta preferimos não reintervir. $O$ caso evoluiu bem, recuperando-se o paciente totalmente. $O$ sangue no leito tumoral foi reabsorvido, como pu Jemos observar em outra TC, após 6 meses.

Caso 6 - J.P.U., 30 anos. Há cerca de 2 anos o paciente apresenta convulsões e cefaléia. A TC feita em outro hospital. evidenciou processo expansivo hiperatenuante parietal direito. A angiografia confirmou o diagnóstico. No ato cirúrgico foi retirado, em bloco, um astrocitoma grau I. com bom plano de clivagem. Após 30 dias, a TC (Fig. 6 A) evidenciou imagem hipoatenuante. $O$ contraste ac'mulou-se na periferia da área. Após 7 meses, nova TC (Fig: 6 B) mostrou molificação do aspecto da área hipodensa e ausência de sinais de recidiva. 

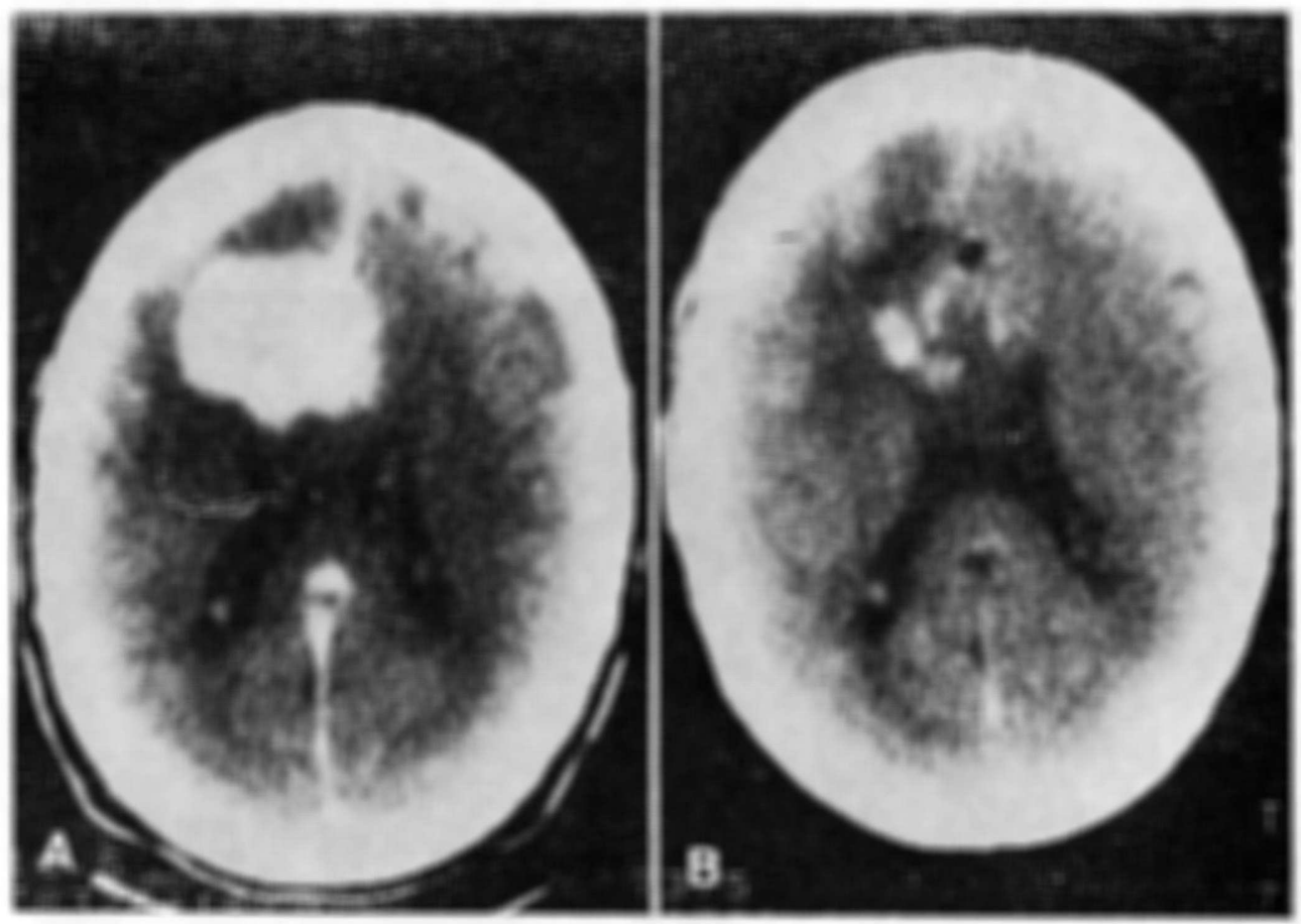

Fig. 5 - Caso 5 . N.M.S.M. Em A. TC com aspecto tipico de meningeoma frontal esquerdo (apos contraste). Em $B$, TC no terceiro dia de pós-operatorio, mostranto sangue

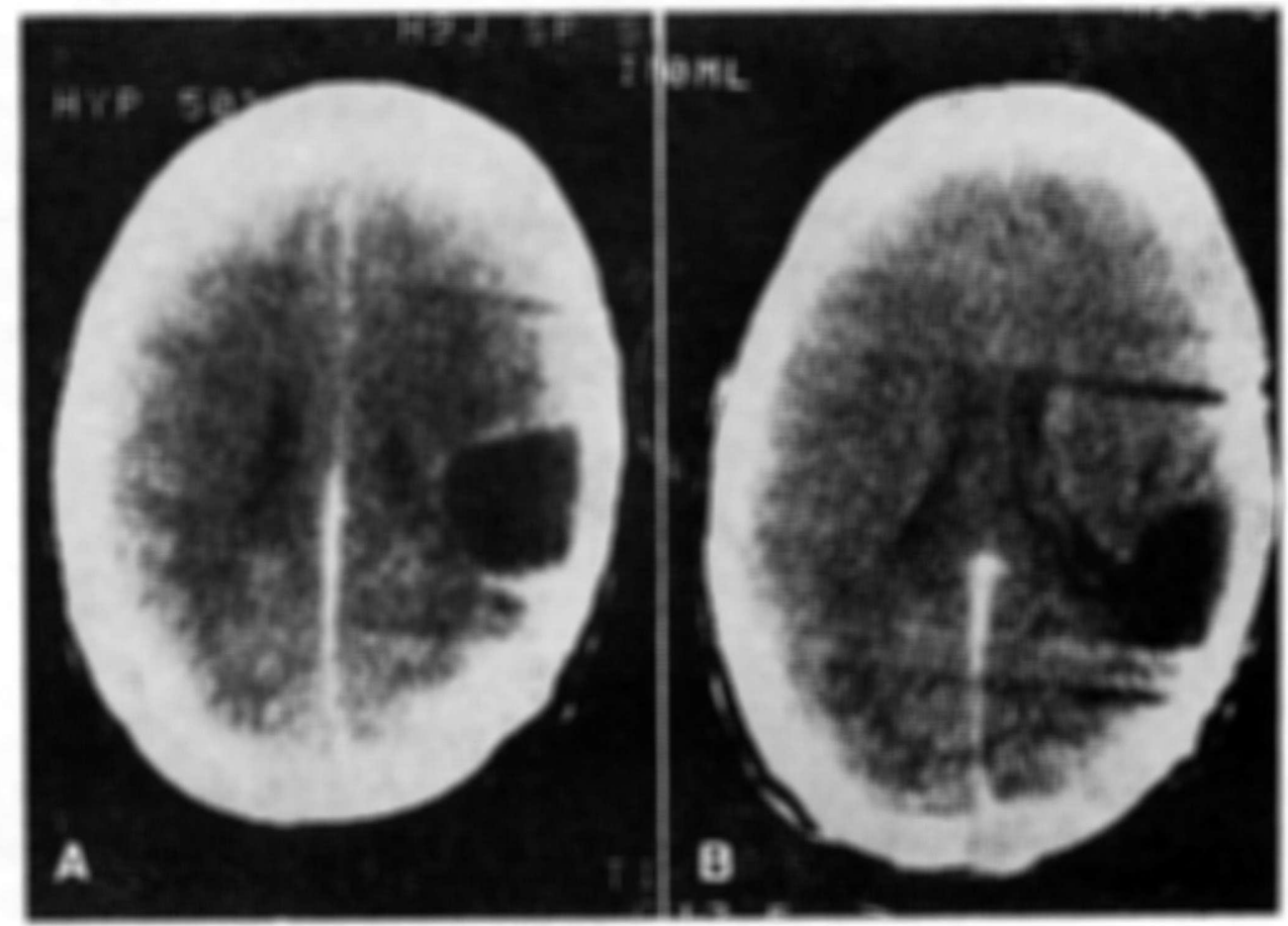

Fig. 6 - Caso 6 . J.P.U. Em A, TC feita 30 dias apos cirurgia de astrocitoma parietal $D$. Em B, TC feita apos 7 meses. 
Caso $y$ - J.L.S., 21 anos. Este paciente apresentava um tuberculoma de fossa posterior (Fig. 7 A) que foi operado. No início evoluiu bem, vindo a piorar no segundo dia. Nova TC (Fig. 7 B) evidenciou hematoma subdural occipital, que foi evacuado cirurgicamente.

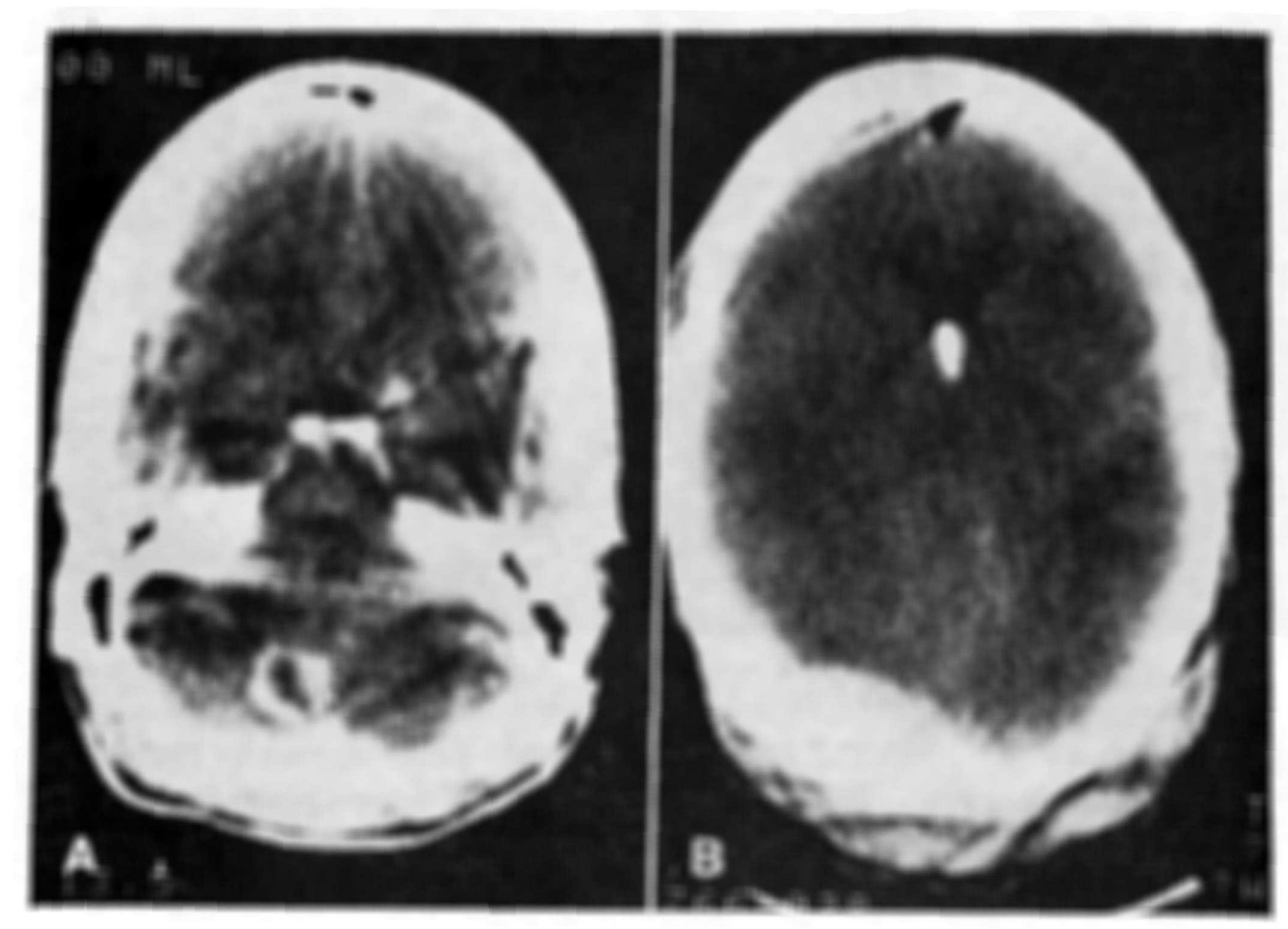

Fig. 7 - Caso 7 . J.L.S. Em A, TC com contraste mostrando tuberculoma de fossa posterior. $\mathrm{Em} B, \mathrm{TC}$ no segundo pós-operatorio evidenciou hematoma occipital esquerdo.

Caso 8 - M.A., 61 anos. Apresentou quadro de cefaléia, vômitos hemiparesia progressiva do lado esquerdo e sonolência, de 2 meses de duração. A TC (Fig. 8 A) mostrou área hipoatenuante parietal direita, com discreta concentração de contraste. A angiografia evidenciou desvios vasculares, sem imagem tumoral. Foi feita biópsia estereotáxica, tendo o exame anátomo-patológico mostrado amolecimento cerebral. Após o ato cirúrgico o doente pioro“, acentuando-se a sonolência e a hemiparesia. No dia. seguinte, nova TC (Fig. 8 B) mostrou que as punções haviam ocasionado sangramento difuso no interior do tecido amolecido. Nova TC, feita após 16 dias, mostrou que o sangue no interior da área de amolecimento havia sido reabsorvido. O caso continuou a evoluir mal, vindo o paciente a falecer 22 dias após a cirurgia.

\section{CONTROLE TARDIO}

No controle tardio tem especial importância a constatação de recidiva tumoral. Também é interessante observar a evolução do hidrocéfalo, de cistos, assim como o efeito da radioterapia e quimioterapia. Uma das maiores dificuldades no acompanhamento dos gliomas é a diferenciação entre radionecrose e recidiva tumoral. Na literatura existem vários trabalhos que mostram o aspecto tomográfico encontrado na radionecrose encefálica ${ }^{1,2,3}$. De maneira geral há lesão 

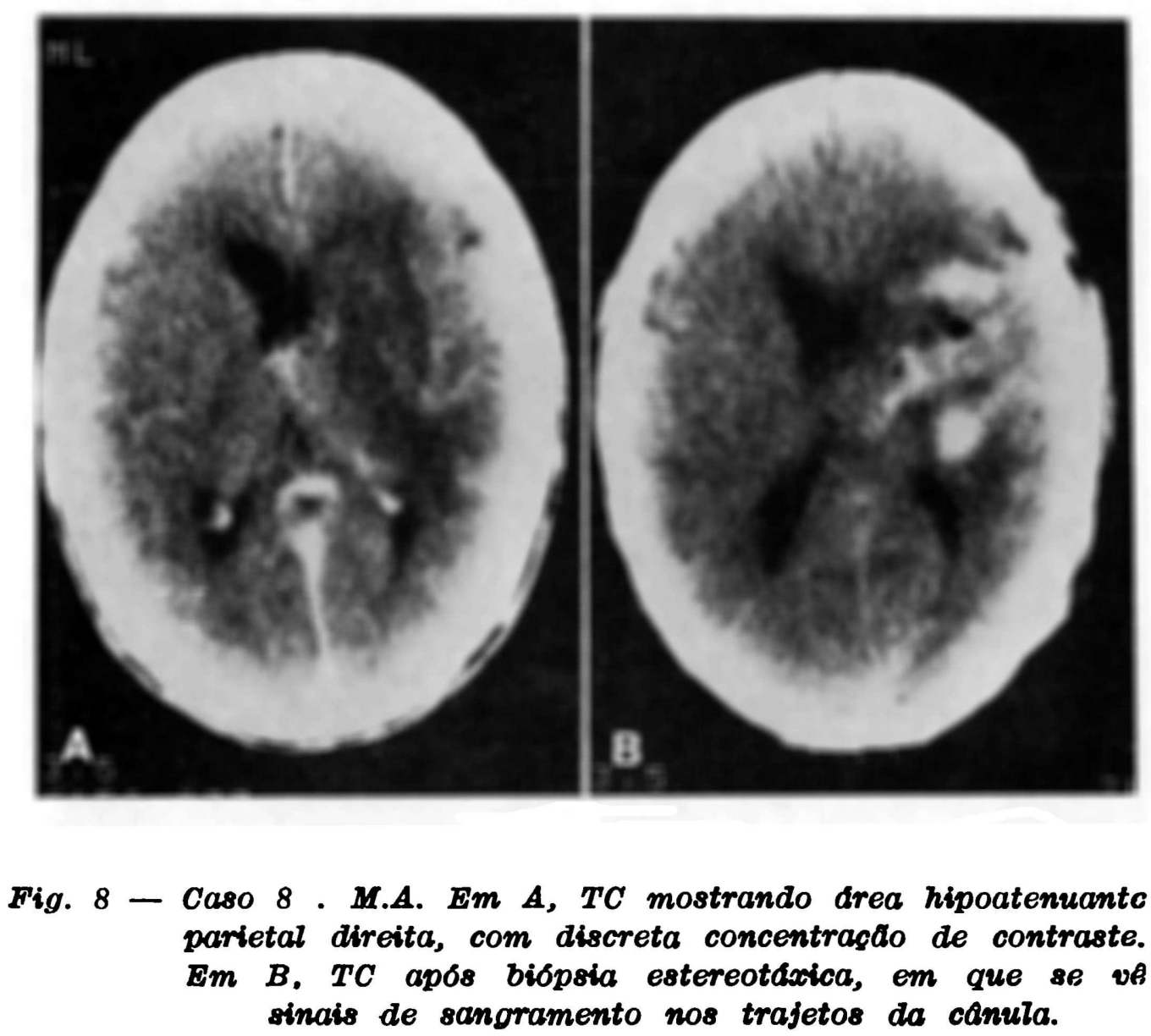

hipoatenuante difusa que concentra irregularmente o contraste iodado. Algumas vezes não se observa captação do contraste. Quando a radionecrose ocorre após irradiação de um tumor intracraniano é difícil fazer o diagnóstico diferencial com recidiva tumoral. $\mathrm{Em}$ alguns pacientes há necessidade de realizar numerosas tomografias para diagnosticar diversas alterações do processo expansivo ou dos ventrículos.

Caso 9 - M.H.N., 8 anos. A menina apresentava quadro de hipertensão intre. craniana e sintrome cerebelar de 2 meses de duração. A TC (Fig. 9 A) mostrou tumor de fossa posterior, hiperaten:-ante, com calcificaçóes e que captava contraste. No ato cirúrgico foi retirado meduloblastoma de vermis e colocado cateter entre III ventriculo e cisterna magna. No pós-operatório formou-se cisto de fossa posterior o uma segunda tomografia evidenciou piora do hidrocéfalo. Indicamos a derivaçăo ventrículo-peritoneal e uma terceira TC (Fig. 9 B )mostrou melhora da dilataça ventricular. Devido a processo infeccioso fomos obrigados a retirar o "shunt", o que determinou piora da hidrocefalia, o que foi comprovado na quarta TC (Fig. 9 C). Tratada a infecção fol refeita a drenagem ventric:.lo-peritoneal e a quinta TC (Fig. 9 D) evidenciou que os ventrículos tinham diminuido. Mais 2 tomografias, realizadas 1 ano e 2 anos após a primelra cirurgla, năo mostraram presença de recidiva.

Caso 10 - N.M. 53 anos. O paciente apresentava quadro progressivo de hipertensăo intracraniana e hemiparesia direita, com 5 meses de duração. A TC (Fig. 10 A) mostrou tumor temporal esquerdo, confirmado pelo estudo angiográfico. Mediante 

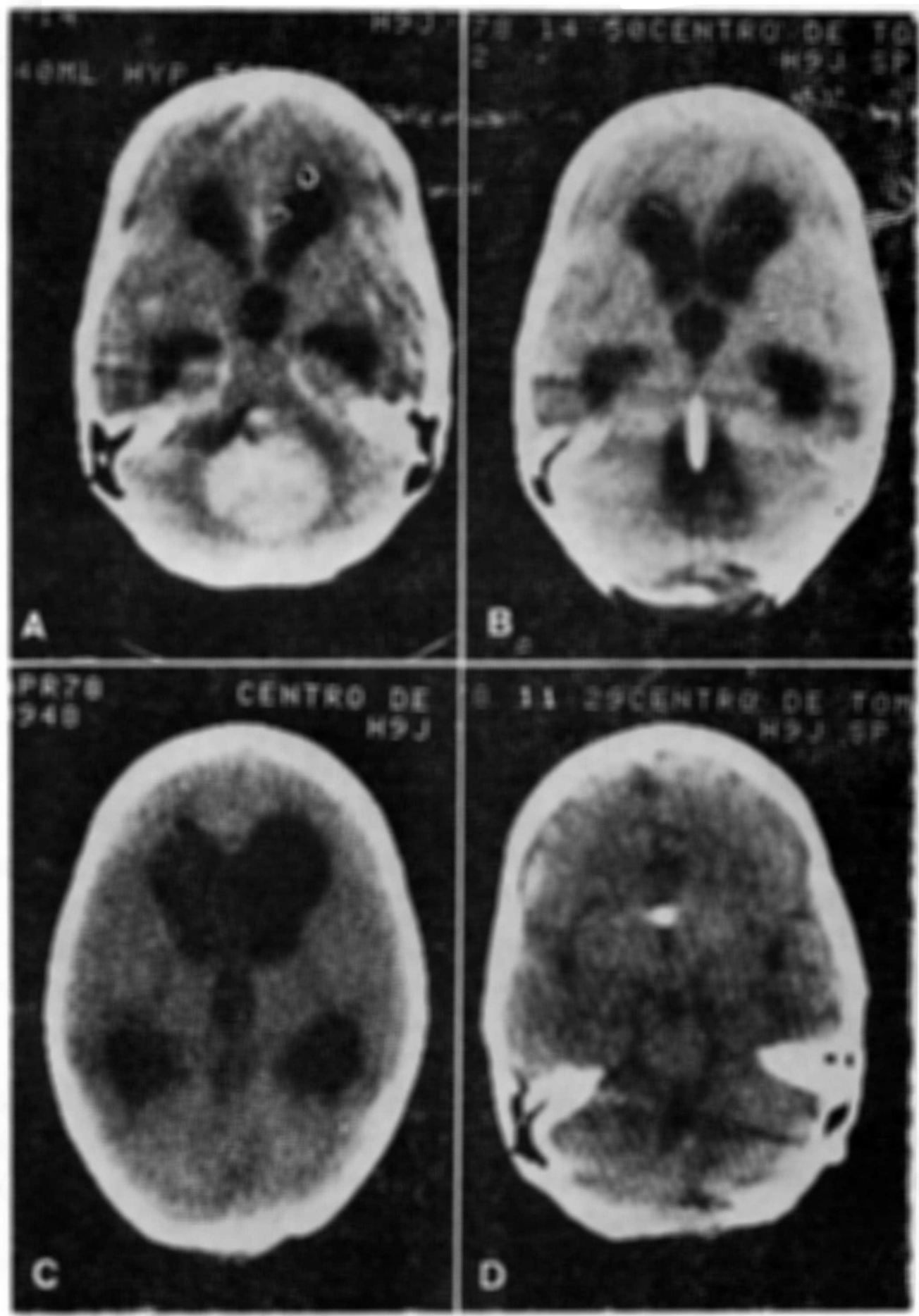

Fig. 9 - Caso 9 . M.H.N. Em A, TC com contraste que diagnosticou o meduloblastoma. Em $B$, observa-se uma hidrocefalia discreta, que se acentuou após retirada da derivação ventrículo-peritoneal (C). Em $D$ nota-se que os ventriculos ficaram colabados, após nova derivacão. 

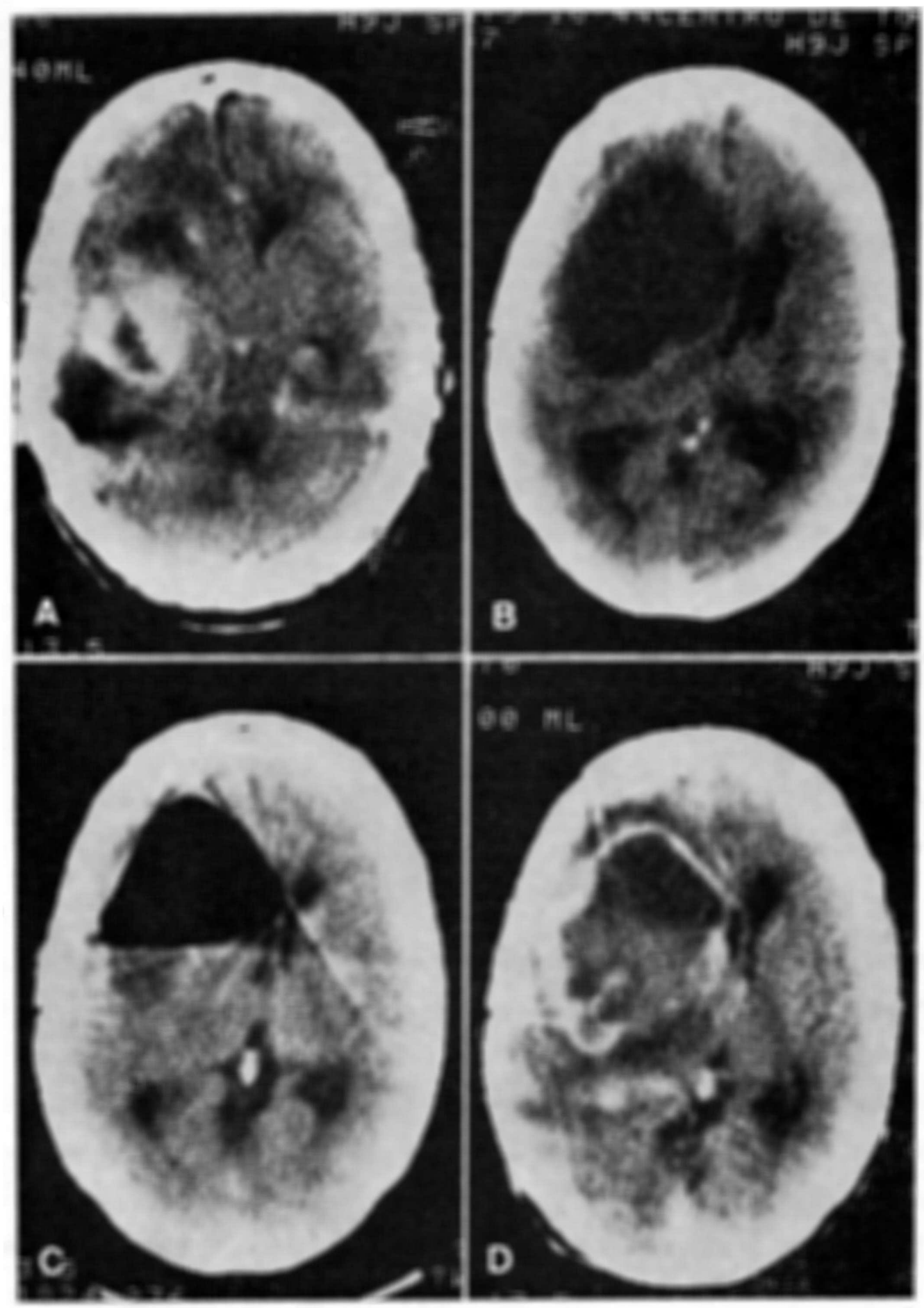

Fig. 10 - Caso 10 . N.M. Em A, TC mostrando o processo expansivo temporal esquerto. $\mathrm{Em} B, T C 3$ meses após o ato cirúrgico, evidenciando grande cisto na área operada. Após punção e esvaziamento, o cisto ficou com grande quantidade de ar (C). Em D, TC mostrando recidiva tumoral, com desaparecimento do cisto. 


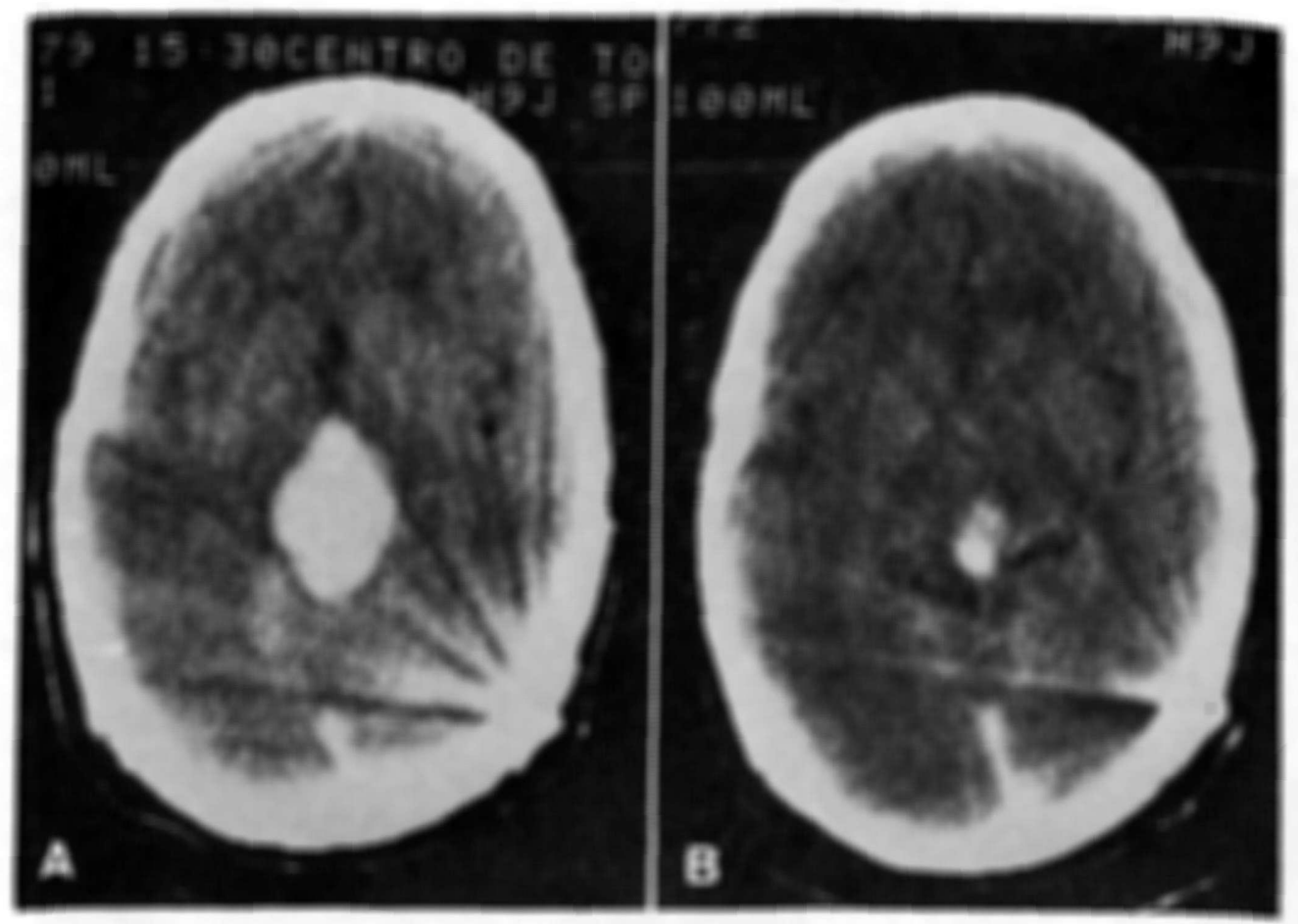

Fig. 11 - Caso 11 . N.P.Fr. Em A, TC com contraste mostrarado tumor da região da pineal, com pequena calcificac̄oo na parte posterior. Apos a radioterapia o tumor praticamente desapareceu, notando-se pequena captasão de contraste próximo à direa calcificada (B)

ato cirúrgico foi feita retirada subtotal de oligodendroglioma. Após 20 dias a segunda TC mostrou área hipoatenuante, sem evidência de processo expansivo. $O$ quadro neurológico piorou após 3 meses (sonolência e aumento da hemiparesia) e a TC (Fig. 10 B) mostrou grande cisto na área operada. No mesmo dia foi feita punção e retirado líquido xantocrômico, com o que houve nítida melhora do quadro neurológico. No dia seguinte, nova TC (Fig. 10 C) mostrou o cisto parcialmente ocupado por ar. O paciente foi submetido a mais 3 punções do cisto, havendo progressivamente maior dificuldade para retirar líquido. Uma ultima TC (Fig. $10 \mathrm{D}$ ) mostrou que o tumor ocupava vasta área, não havenjo parte cística.

Caso 11 - N.P.F., 23 anos. Paciente com quadro progressivo de hipertensão intracrantana. O estudo angiográfico, feito em outra clínica, mostrou dilatação ventricular, tendo sido realizzada derivação ventrículo-peritoneal. A TC (Fig. 11 A) mostrou ventrículos colabados e tumor na região da pineal, ao lado de pequena área calcificada. F'oi feito tratamento radioterápico e, 35 dias após a primeira TC, a repetição do exame mostrou apenas pequena área de retenção de contraste (Fig. 11 B), na frente da calcificação. 


\section{RESUMO}

A tomografia computadorizada tem grande valor no controle dos pacientes com tumores cerebrais. Imediatamente após o ato cirúrgico pode ser útil para avaliar a retirada do processo expansivo ou para comprovar a área atingida. Nos primeiros dias após a intervenção tem sido indicada principalmente quando há piora do quadro neurológico. Nestas condições, o exame permite verificar a existência de ar, edema, hematoma, desvios ventriculares e hidrocéfalo. Os dados fornecidos são mais precisos que os obtidos com outros exames neurorradiológicos ou com a medida contínua da pressão intracraniana, métodos menos inócuos. A longo prazo a tomografia permite o diagnóstico precoce de recidivas, a avaliação da eficácia de tratamentos radio ou quimioterápicos e o controle do hidrocéfalo. Ainda não é possível fazer o diagnóstico diferencial entre radionecrose e recidiva tumoral.

SUMMARY

Computed tomography in the follow-up of brain tumors.

Computed Tomography (CT) has been very useful in the follow-up of patients with brain tumors. Soon after the craniotomy, CT would show any residual tumor if an incomplete excision was done. CT has been essential when one needs to evalute any deterioration in the patient's neurological status post operatively. In such instances, CT may detect brain edema, hematoma, hydrocephalus and the presence of air. CT findings are more accurate than those obtained from conventional neuroradiology or from intracranial pressure monitoring, being also more innocuous than those methods. Later in the post operative follow-up, CT allows, besides precocious diagnosis of tumor recurrences, a good evaluation of the results obtained by chemiotherapy or radiotherapy. When hydrocephalus is accompanieing the primary pathology, ventricular size can be rapidly evaluated by $C T$. Difficulties may arise when one wishes to differentiate $\mathrm{C}^{\prime} \mathrm{l}$ ' images of radionecrosis from tumor recurrences at least in the initial stages.

\section{REFERENCIAS}

1. DIENGDOH, J. V. \& BOOTH, A. R. - Postirradiation necrosis of the temporal lobe presenting as a glioma. J. Ne rosurg. 44:732, 1976.

2. MARTINS, A. N.; JOHNSTON, J. S.; HENRY, J. M.; STOFFEL, T. J. \& DI CHIRO, G. - Delayed radiation necrosis of the brain. J. Neurosurg. 47:336, 1977.

3. QUEIROZ, L. S. \& CRUZ NETO, J. N. - Late pseudotumoral brain necrosis following irradiation of a scalp neoplasm. J. Neurosurg. 45:581, 1976.

Clinica Neurocirúrgica e Centro de Tomografia do Hospital 9 de Julho - Rua Peixoto Gomide 625 - 01409 são Paulo, SP - Brasil. 